\title{
Synergistic interactions between two distinct elements of a regulated splicing enhancer
}

\author{
Kristen W. Lynch and Tom Maniatis \\ Department of Molecular and Cellular Biology, Harvard University, Cambridge, Massachusetts 02138 USA
}

\begin{abstract}
Regulated alternative splicing of doublesex (dsx) pre-mRNA requires a splicing enhancer designated the $d s x$ repeat element (dsxRE) that contains six copies of a 13-nucleotide repeat sequence. Previous studies have shown that the activity of the $d s x R E$ requires the splicing regulators Transformer (Tra) and Transformer 2 (Tra2), and one or more members of the SR family of general splicing factors. In this paper we identify a purine-rich enhancer (PRE) sequence within the $d s x R E$, and show that this element functionally synergizes with the repeat sequences. In vitro binding studies show that the PRE is required for specific binding of Tra2 to the $d s x R E$, and that Tra and SR proteins bind cooperatively to the $d s x R E$ in the presence or absence of the PRE. Thus positive control of $d s x$ pre-mRNA splicing requires the Tra- and Tra2-dependent assembly of a multiprotein complex on at least two distinct enhancer elements.
\end{abstract}

[Key Words: Alternative splicing; doublesex; sex determination; transformer proteins]

Received November 23, 1994; revised version accepted December 21, 1994.

A well-characterized example of the regulation of alternative pre-mRNA splicing is the sex determination pathway in Drosophila melanogaster (for review, see Baker 1989; Stcinmann-Zwicky et al. 1990; Maniatis 1991; Rio 1992). In the final step of this pathway the female-specific Transformer (Tra) protein, together with Transformer 2 (Tra2), a protein expressed in both males and females, positively controls the alternative splicing of doublesex $(d s x)$ pre-mRNA (Nagoshi ct al. 1988; McKcown et al. 1988). The male (default) splicing of $d s x$ premRNA produces an mRNA consisting of exons $1-3,5$, and 6 , encoding a transcription factor required for male sexual differentiation. Conversely, the female (regulated) pattern of $d s x$ pre-mRNA splicing results in an mRNA containing exons $1-4$, which encodes a transcription factor required for female sexual differentiation (Burtis and Baker 1989; Fig. 1A).

The Tra/Tra2 regulation of $d s x$ pre-mRNA splicing provides a model for understanding positive control of alternative splicing. The female-specific $3^{\prime}$ splice site immediately upstream of the fourth exon deviates significantly from the consensus sequence and thus in males is not recognized by the splicing machinery (Burtis and Baker 1989). Use of this splice site in females requires the proteins Tra and Tra2 as well as a regulatory element located within the fourth exon (Hedley and Maniatis 1991; Hoshijima et al. 1991; Ryner and Baker 1991; Tian and Maniatis 1992). This regulatory element, located 300 nucleotides downstream of the female-specific $3^{\prime}$ splice site, is characterized by the presence of six 13-nucleotide repeat sequences (see Fig. $1 \mathrm{~B}$ ), and hence is referred to as the $d s x$ repeat element (dsx RE) (Burtis and Baker 1989; Nagoshi and Baker 1990; Tian and Maniatis 1993).

Tra2 binds specifically to the $d s x \mathrm{RE}$ (Hedley and Maniatis 1991), and both Tra and Tra2 are found in a multiprotein complex assembled on the $d s x \mathrm{RE}$ in vitro (Tian and Maniatis 1992; 1993). This complex also contains SR proteins (Tian and Maniatis 1993), a family of general splicing factors characterized by the presence of an RNAbinding domain (RNP domain) and a serine/arginine (SR)-rich region (for review, see Lamm and Lamond 1993; Horowitz and Krainer 1994|. Similar to the SR proteins, Tra2 contains both an RNP and an SR domain, whereas Tra contains only an SR domain (Boggs et al. 1987; Amrein et al. 1988). The SR domain is required for the in vitro splicing activity of SR proteins /Caceres and Krainer 1993; Zuo and Manley 1993), and it has been implicated in both protein-protein interactions and subnuclear localization (Krainer et al. 1991; Fu and Maniatis 1992; Zahler et al. 1992; Wu and Maniatis 1993).

The involvement of SR proteins in the regulation of $d s x$ female-specific splicing was directly demonstrated by in vitro splicing studies showing that SR proteins are required, in addition to $\mathrm{Tra}$ and $\mathrm{Tra} 2$, to form a functional complex that commits $d s x$ pre-mRNA to the female-specific pattern of splicing (Tian and Maniatis 1993). Protein-protein interaction studies revealed that Tra and Tra2 interact with each other, with SR proteins, and with other general splicing factors (Wu and Maniatis 1993; Amrein et al. 1994). These observations suggest that the multiprotein complex assembled on the $d s x \mathrm{RE}$ 
A
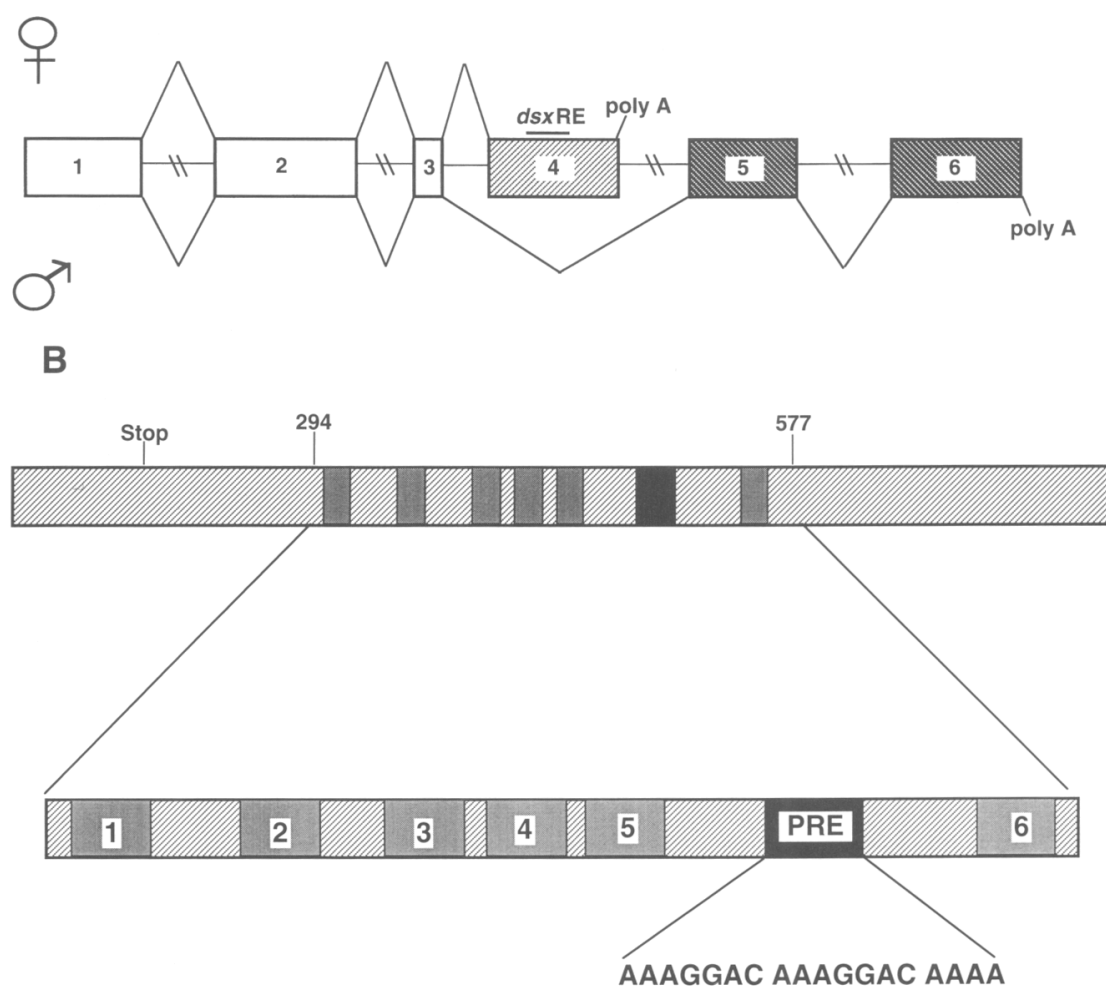

Repeat Consensus: UCUUCAAUCAACA
Figure 1. The sex-specific pattern of $d s x$ pre-mRNA alternative splicing and the $d s x$ splicing enhancer ( $d s x \mathrm{RE})$. (A) Open regions represent common exons, the light hatched region (exon 4) is the female-specific exon, and dark hatched regions (exons 5 and 6 ) are male-specific exons. Lines between the boxes represent introns, and the lines above and below the pre-mRNA illustrate the female- and male-specific patterns of alternative splicing. Sites of cleavage and polyadenylation are labeled poly A. (B) Enlargement of the female-specific exon 4 showing the organization of the $d s x$ RE. Numbers above the fourth exon indicate the number of nucleotides from the beginning of the exon. Numbered regions indicate the positions of the repeat sequences, with the consensus sequence shown in the box below. The region labeled PRE indicates the position of the purine-rich region with the sequence indicated below. facilitates the assembly of spliceosomal components on the weak female-specific 3' splice site through a network of protein-protein interactions (Tian and Maniatis 1993; Wu and Maniatis 1993; Amrein et al. 1994). The ability of the $d s x$ RE to activate heterologous $3^{\prime}$ splice sites led to the proposal that the $d s x \mathrm{RE}$ is a regulated splicing enhancer, similar in function to transcriptional enhancers (Tian and Maniatis 1993).

Constitutive splicing enhancers have been identified and characterized in a number of pre-mRNAs. These enhancers are located within exon sequences located downstream from introns containing a weak 3' splice site (Watakabe et al. 1993; Xu et al. 1993; Lavigueur et al. 1993; Tanaka et al. 1994) and, in one case, a weak 5' splice site (Dirksen et al. 1994; Sun et al. 1993a,b). A characteristic feature of these enhancers is that they are rich in purines and, similar to the $d s x \mathrm{RE}$, they are specifically recognized by SR proteins and U1 snRNP (Lavigueur et al. 1993; Sun et al. 1993b; Staknis and Reed 1994). However, there are two important differences between the purine-rich and $d s x$ splicing enhancers. First, the purine-rich enhancers are constitutively active, whereas the $d s \times \mathrm{RE}$ requires the regulatory proteins Tra and Tra2. Second, purine-rich enhancers must be located within 100 nucleotides of the regulated intron, whereas the $d s x R E$ can function at least 500 nucleotides away (Tian and Maniatis 1994). Significantly, however, the $d s \times R E$ can function as a constitutive enhancer when located within 100 nucleotides of the regulated intron (Tian and Maniatis 1994).

The six 13-nucleotide repeat sequences in the $d s x \mathrm{RE}$ bear little resemblance to purine-rich enhancers. The consensus sequence of the $d s x$ repeat is UCUUCAAUCAACA, whereas a comparison of various purine-rich sequences (Tanaka et al. 1994) reveals a common motif of GAAGGA. A closer examination of the $d s x$ RE, however, revealed a previously unnoticed near-consensus purine-rich sequence between repeats 5 and 6 (Fig. 1B). Thus the $d s x R E$ may consist of two types of regulatory elements, the repeat sequences R 1-6, and the purine-rich enhancer (PRE). In this paper we investigate the role of these two sequences in the function of the $d s \times R E$. We show that either the R1-5 or the PRE alone can constitutively activate splicing when located near the intron, but both are required for efficient Tra/Tra2-dependent activation of female-specific splicing at a distance. In vitro binding studies with recombinant proteins show that both the repeats R1-5 and the PRE are required for 
specific binding of Tra, Tra2 and SR proteins. We conclude that the $d s x \mathrm{RE}$ splicing enhancer complex is assembled through cooperative interactions between Tra, Tra2 and the SR proteins, and that these interactions require both the repeat sequences and the PRE.

\section{Results}

The dsx repeats $R 1-5$ and the PRE are distinct constitutive splicing enhancer elements

The PRE includes two direct repeats of the sequence AAAGGAC, which differs at only one position from the GAAGGAC sequence found in the IgM M2 exon, the bGH exon 5 and many other purine-rich enhancers (Tanaka et al. 1994). We therefore investigated the role of the PRE in the constitutive and regulated activities of the $d s x$ splicing enhancer. The constitutive splicing activity was assayed by placing the $d s x \mathrm{RE}$ containing or lacking the PRE within 100 nucleotides of the femalespecific 3' splice site. The role of the PRE in the Tra- and Tra2-dependent activity of the $d s x$ RE was examined by placing the same elements 300 nucleotides downstream (Tian and Maniatis 1994).

As shown in Figure 2 the $d s \times R E$ sequence containing both the repeats R2-5 and the PRE can function as a splicing enhancer in the absence of Tra and Tra2 when positioned close to the regulated intron (R2-5PRE; lanes 1-3). However, either the repeat sequences alone (R2-5; lanes 4-6), a synthetic repeat sequence (R2x4; lanes 7-9) or a single copy of the PRE (dsxPRE; lanes 10-12) can constitutively activate the weak $3^{\prime}$ splice site as efficiently as the intact $d s x \mathrm{RE}$. We note that the presence of the PRE alone (dsxPRE) appears to stabilize this RNA during incubation in nuclear extract. As expected, little splicing is observed in the absence of any enhancer sequences $(d s \times \Delta \mathrm{E}$; lanes $13-15)$, and the addition of Tra and Tra2 does not further stimulate the constitutive activation of splicing (Fig. 2, lanes 3,6,9,12,15; Tian and Maniatis 1994). We conclude that the $d s x \mathrm{RE}$ contains at least two distinct splicing enhancer elements that can function constitutively when positioned close to the $d s x$ female-specific $3^{\prime}$ splice site.

Both the dsx repeats $R 1-5$ and the PRE are required for Tra-and Tra2-dependent splicing enhancer activity

In marked contrast to the constitutive enhancer activities described above, neither four repeat sequences (R2-5 and R2x4; Fig. 3A, lanes 10-12,13-15) nor the PRE (dsxPRE; lanes 16-18) are sufficient to activate splicing when moved to their natural position 300 nucleotides downstream of the regulated 3' splice site, even in the presence of Tra and Tra2. Instead, both the $d s x$ repeats and the PRE are required for efficient Tra/Tra2-dependent activation of femalc-specific splicing.

When five repeats are positioned at their wild-type spacing from the female-specific $3^{\prime}$ splice site, deletion of the purine-rich element causes a significant decrease
Figure 2. Either the $d s x$ repeat sequences or the PRE alone can function as constitutive splicing enhancers. As indicated by the schematic below the autoradiograph, all of the $d s x$ RNA substrates contain part of exon 3 , the entire regulated intron, and 100 nucleotides of exon 4 . The substrates differ from each other by the presence or absence of different regions of the $d s x R E$. (R2-5PRE) The $d s x$ RE lacking repeats 1 and 6 ; (R2-5) the $d s \times \mathrm{RE}$ lacking repeats 1 and 6 and the PRE; $(\mathrm{R} 2 \times 4)$ a synthetic enhancer consisting of four copies of repeat $2 ;(d s x$. PRE) the PRE alone; (ds $\triangle \Delta E)$ the RNA substrate without the $d s \times R E$. Splicing intermediates were not detected because the splicing reactions were incubated for $2 \mathrm{hr}$. (NE) Nuclear extract; (- and + / pre-mRNA analyzed before or after the splicing reaction, respectively. Approximately onetenth as much RNA was loaded in the NE - lanes as was used in the splicing reactions. Tra + Tra2 + and - indicate the presence or absence of these proteins, respectively, in the splicing reactions. The band directly below the precursor in lanes 11 and 12 is presumably a lariat intermediate. Arrowheads indicate spliced product.

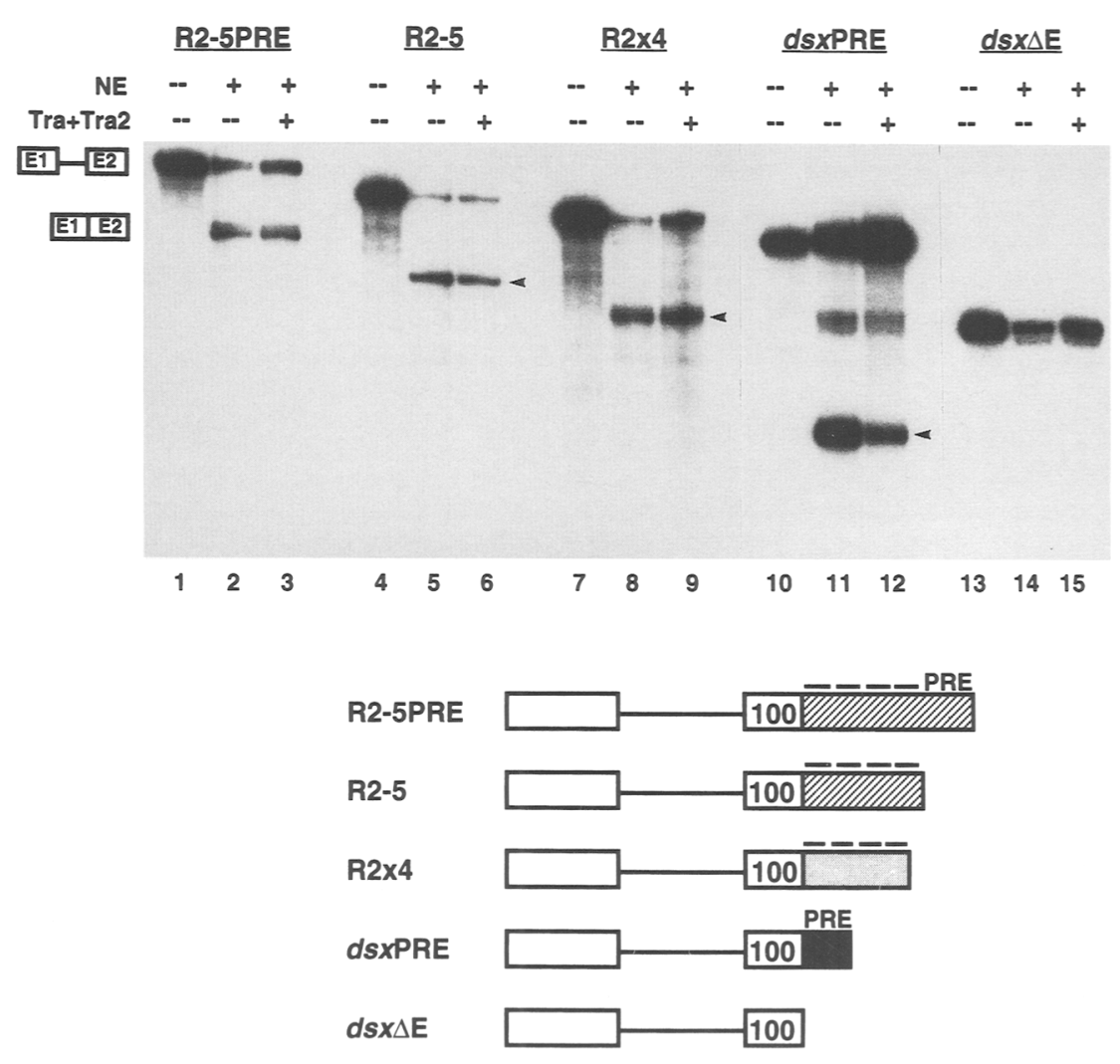


A

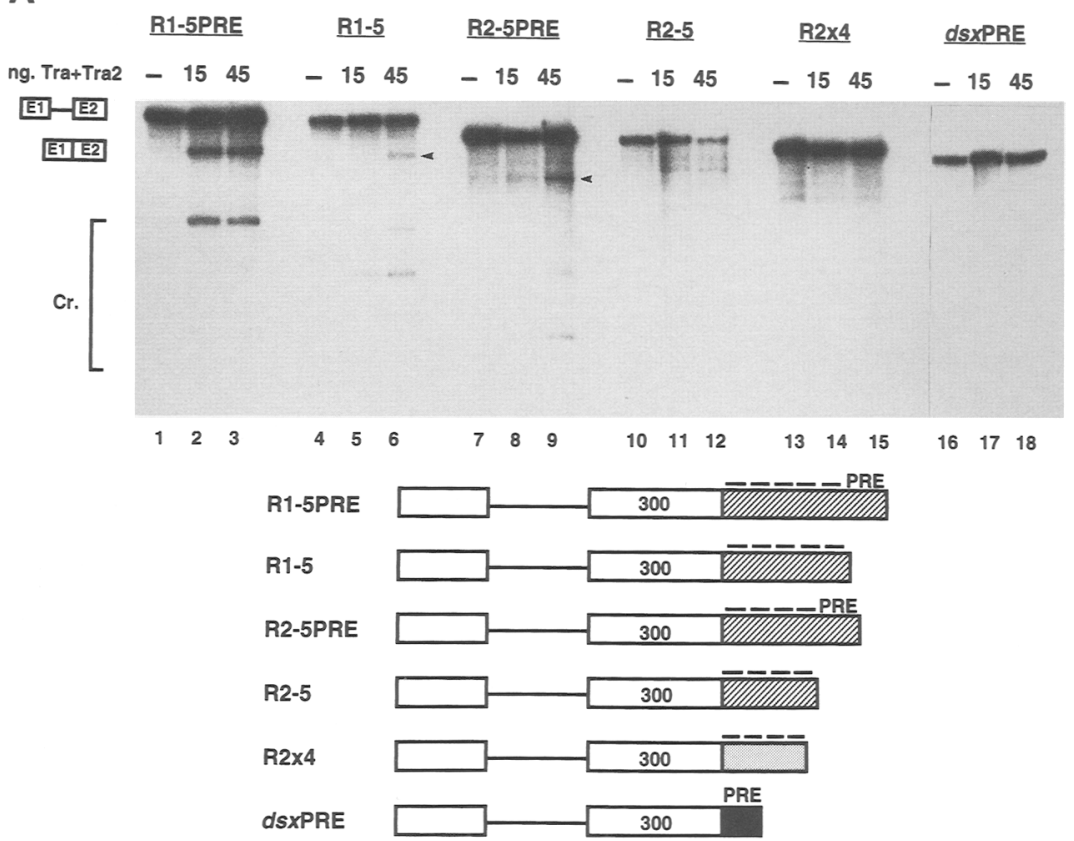

B
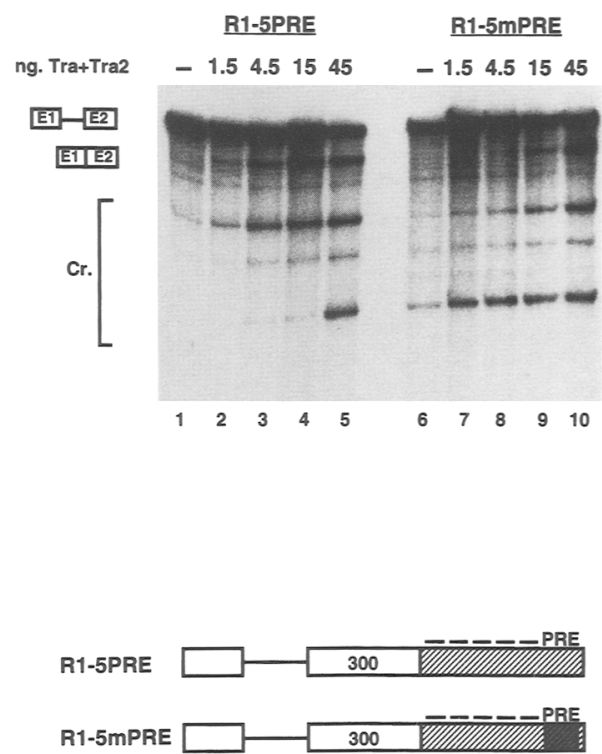

Figure 3. Both the repeat sequences and the PRE are required for efficient Tra/Tra2-dependent activation of splicing. (A) As indicated by the schematics below the autoradiograph, the $d s x$ RNA substrates are similar to those shown in Fig. 2 except that the $d s x$ RE segments are located 300 nucleotides downstream from the 3' splice site. (R1-5PRE) The dsxRE lacking only repeat 6; (R1-5) the $d s \times R E$ lacking repeat 6 and the PRE. The indicated nanograms of Tra and Tra 2 added to reaction are approximations based on Bradford assays. Spliced products resulting from the use of cryptic splice sites are labeled $\mathrm{Cr}$. Arrowheads indicate correctly spliced product. $(B)$ In vitro splicing reactions carried out with constructs containing either a wild type or mutant PRE. R1-5mPRE contains six point mutations in the PRE, thus changing the sequence from AAAGGACA_AAGGACAAAA to AATCGACCATCGACCAAA, with underlined residues indicating sites of mutations.

in the efficiency of splicing, especially at low concentrations of Tra/Tra2 (Fig. 3A; compare lanes 2 and 5). Consistent with previous results, splicing efficiency also decreases when the number of repeats is reduced from five to four in the presence of the PRE (R1-5PRE; Fig. 3A, lanes 1-3; compare to R2-5PRE, lanes 7-9). However, in the absence of the PRE, four repeats in their native context (R2-5; Fig. 3A, lanes 10-12) or as synthetic repeats (R2 24 , lanes 13-15) are essentially inactive even in the presence of high levels of Tra/Tra2. We conclude that synergistic interactions between the repeat sequences and PRE are required for the Tra- and Tra2-dependent activity of the $d s x$ RE.

A significant loss of activation efficiency was observed when the PRE was deleted. To determine whether the purine content of the PRE is required for its activity we introduced multiple base substitutions into the PRE /see legend to Fig. 3B). Pre-mRNA containing base substitutions in the PRE (Fig. 3B, R1-5mPRE, lanes 6-10) requires three- to fivefold more Tra and Tra2 than the wild-type RNA substrate (R1-5PRE, lanes 1-5; compare lanes 2 and 3 with 8 and 9 , respectively) to achieve the same level of splicing activity. The failure of these base substitutions to completely inactivate the enhancer is likely because the primarily cytosine-substituted sequence has residual constitutive splicing enhancer activity (Tanaka et al. 1994). This possibility is consistent with the observation that a nonpurine-rich sequence can function as a constitutive enhancer, albeit less well than the purine-rich sequence (Staknis and Reed 1994).

Deletion of the PRE decreases the affinity of Tra2 and $S F 2$, but not SC35, for the dsxRE

To investigate the mechanism of the synergistic interactions between the $d s x$ repeat sequences and the PRE we carried out in vitro nitrocellulose binding experiments. Previous studies demonstrated that Tra and Tra2 along with SR proteins (namely SC35 and SRp55) are sufficient to form a functional regulatory complex on the $d s x$ RE (Tian and Maniatis 1993). We therefore studied the binding of purified recombinant Tra, Tra2, SC35 and SRp55, as well as SF2/ASF, to the $d s \times R E$ containing or lacking the PRE. It is important to note that these RNAs do not contain splice sites. Thus we do not address the question of how these proteins facilitate enhancer- $3^{\prime}$ splice site interactions.

Figure 4 shows the binding curves obtained with each protein and RNAs containing repeats $2-6(R 2-6)$, repeats 2-5 including the PRE (R2-5PRE), or repeats 2-5 lacking the PRE (R2-5). The affinity of Tra2 and SF2/ASF for R2-6 and R2-5PRE, which differ only in the number of repeats, is very similar. However, the affinity of both of these proteins drops by four- to fivefold upon deletion of 

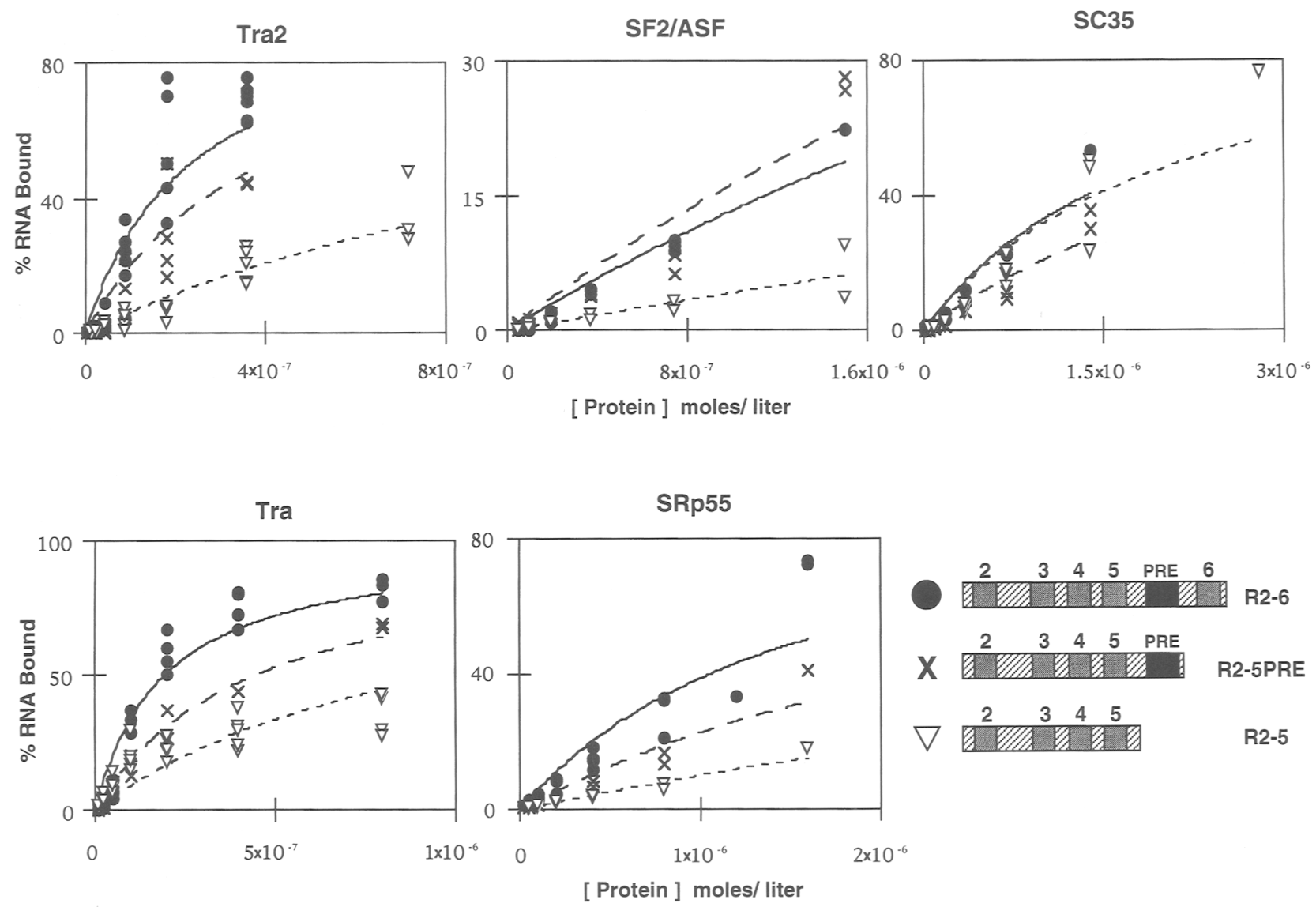

Figure 4. Tra2 and SF2/ASF, but not SC35, require the presence of the PRE for efficient binding to the dsxRE. Graphs showing the results of nitrocellulose filter-binding experiments in which the percentage of labeled RNA retained on a filter is plotted against the amount of protein added to the binding reaction. The protein concentration is varied, whereas RNA is held constant at a concentration of $\sim \mathrm{l} \mathrm{nM}$. The horizontal axis is protein concentration (molarity, $\mathrm{M}$ ) as approximated by Bradford assays. The vertical axis is percent of RNA bound, which is measured as cpm retained on nitrocellulose filters relative to total cpm RNA in the binding reaction, minus $\mathrm{cpm}$ retained on filters in the absence of protein $(<3 \%$ of input RNA). Binding reactions and filtration procedure are as described in Material and methods. and solid lines correspond to the RNA R2-6; X and large dashed lines correspond to R2-5PRE RNA; $\nabla$ and small dashed lines correspond to R2-5. Data were fitted to the standard binding equation $\left.y=\mid 100^{*} x\right) /\left(\max _{1 / 2}+x\right)_{\text {, which is }}$ based on the Michaelis-Menton formula.

Figure 5. Binding specificities of Tra, Tra2, and SR proteins for the $d s x$ RE. $(A)$ Shown are average binding specificities derived from at least three independent experiments, except for SF2/ASF or SRp55 alone, which were based on two independent experiments. Because of inherent error, standard deviations of all reported averages are between 10 and $50 \%$; therefore, all differences of at least twofold are considered significant. N/D notation indicates that the specificity of SF2 could not be measured because of the low binding affinity of this protein. $(B)$ Representative data used in the calculation of binding specificities. The horizontal axis is RNA competitor concentration $(\mathrm{M})$. The vertical axis is percent RNA bound to filter, relative to a reaction lacking any competitor. ( $\downarrow$ and dashed lines) Nonspecific competitor; ( $\square$ and solid lines) specific competitor. Binding reactions are carried out in the presence of increasing amounts of specific or nonspecific competitor. In each experiment the specific competitor is identical to the probe, and the nonspecific competitor is a transcript of comparable length from a region of the fourth exon downstream of the $d s x$ RE. This nonspecific competitor is the same as used in previous studies (D6 construct in Tian and Maniatis 1992, 1993). Probe and competitor are mixed before protein is added. When more than one protein is present in a reaction, proteins are also mixed prior to adding to probe and competitor. Inhibiting concentration $(50 \%)\left(\mathrm{IC}_{50}\right)$ for each competitor is calculated as the concentration of competitor needed to compete away $50 \%$ of binding, relative to a reaction in which no competitor is added. The calculated specificity is equal to IC $_{50}$ (nonspecific) divided by $\mathrm{IC}_{50}$ (specific). We note that for SC35 alone a vast excess of specific competitor relative to probe is necessary for $50 \%$ inhibition of binding. We do not understand the reason for this but suggest that it may be a consequence of the tendency of SR proteins to aggregate, and of the low specificity of SC35 binding. For example, aggregates of SC35 may be formed on individual labeled molecules, and the competitor would have to dissociate the aggregate before competing with the binding of the labeled RNA. We emphasize that the calculated binding specificities are derived from internal comparisons of the $\mathrm{IC}_{50} \mathrm{~s}$ within a single experiment, and that because of variations between experiments meaningful comparisons cannot be made from isolated $\mathrm{IC}_{50}$ values between individual experiments. $(C)$ Representative data used in the calculation of specificity of Tra2 binding to the R2-5PRE and R2-5 RNA probes. Competition experiments were performed and analyzed as described in $B$. 

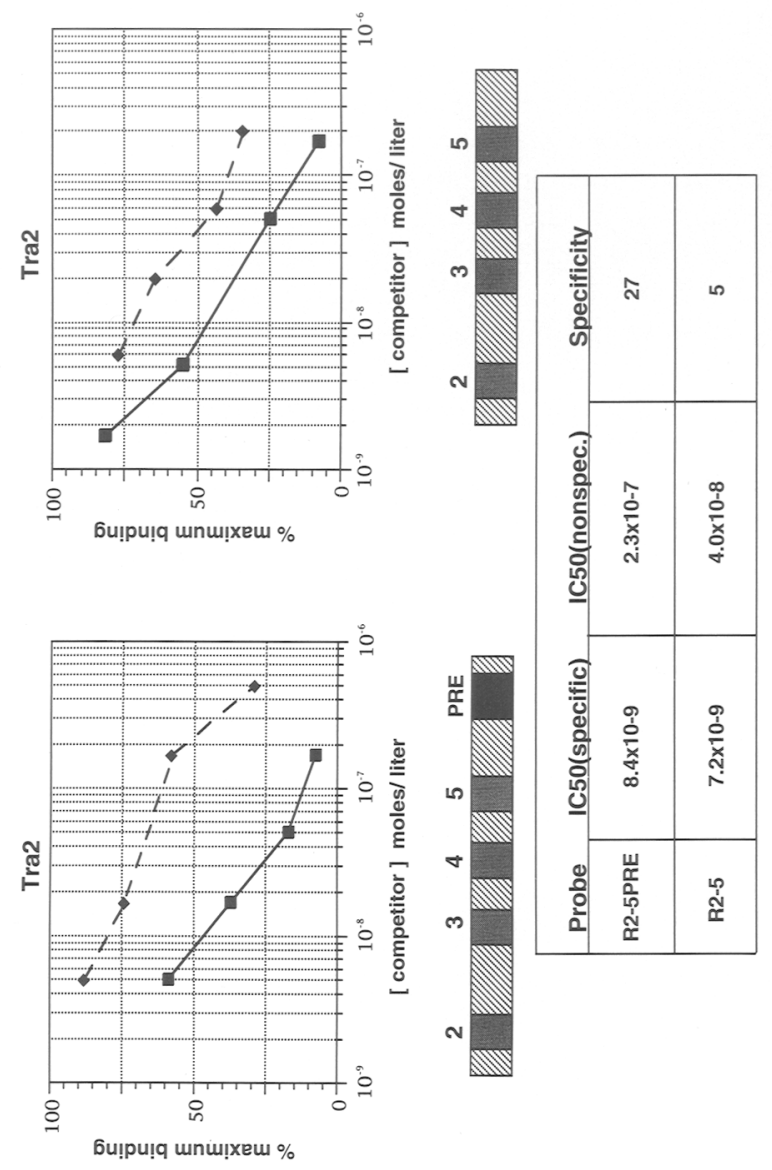

0

赵
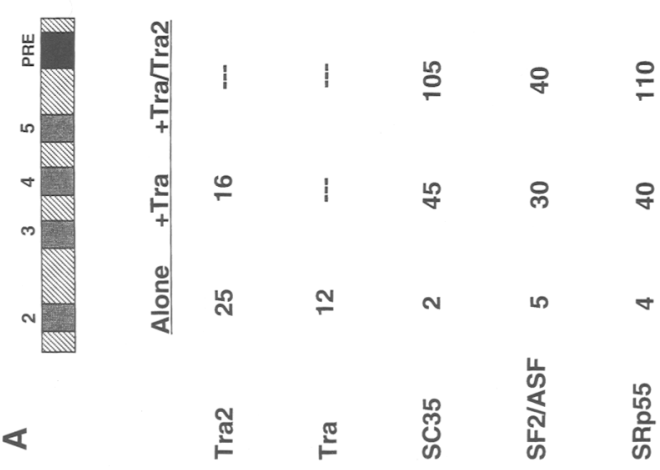

《 $\quad$ ॠ
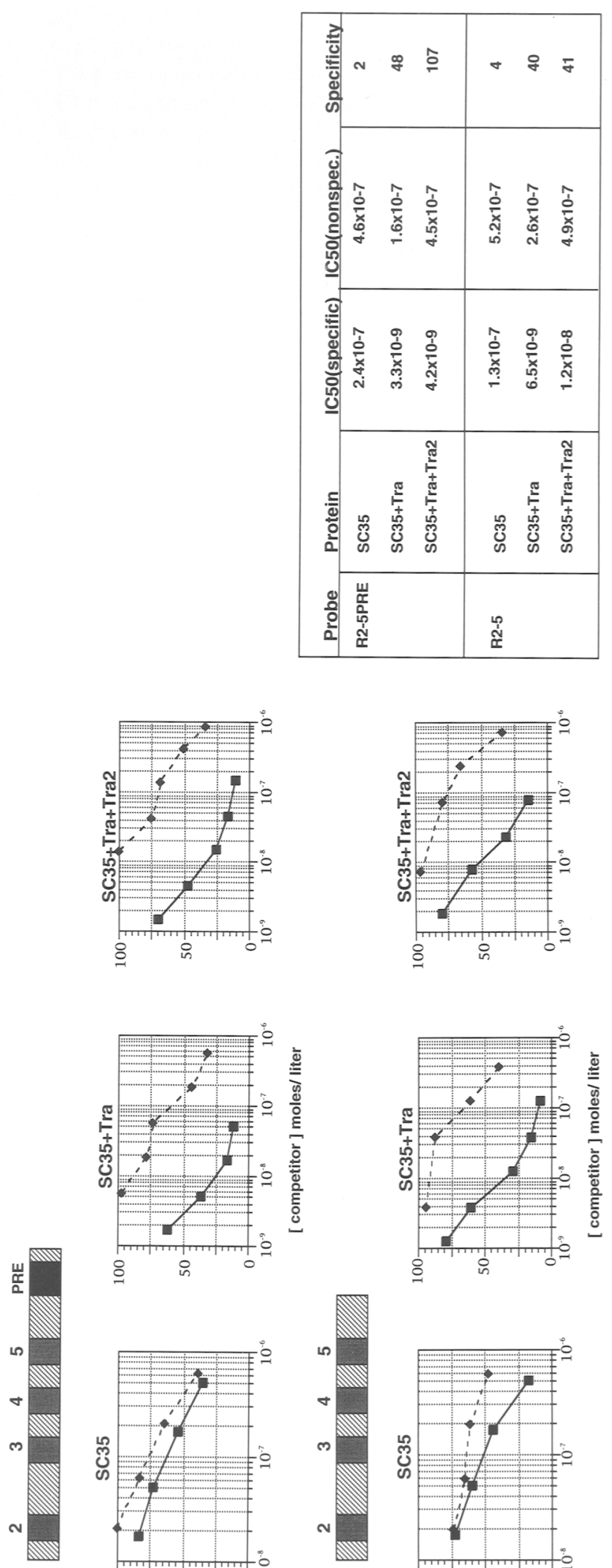
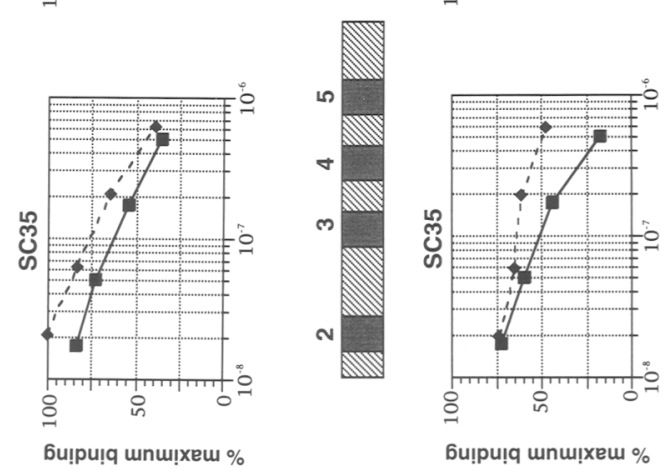

6u!̣pu!̣ unw!̣xem \% 
the purine-rich region (difference between R2-5PRE and $\mathrm{R} 2-5$ ). In contrast, none of the deletions affect the binding of SC35 to the $d s x \mathrm{RE}$. Thus, the repeat sequences alone are sufficient for SC35 binding whereas wild-type levels of binding of Tra2 and SF2/ASF require the PRE.

The relative affinities of Tra and SRp55 for the $d s x R E$ decrease slightly (twofold) upon deletion of either a repeat sequence (compare R2-6 with R2-5PRE) or the purine-rich region (compare R2-5PRE with R2-5). This decrease in affinity could be a nonspecific effect resulting from changes in RNA secondary structure. Alternatively, the binding of Tra and SRp55 to the $d s \times R E$ may be mediated through both the repeat sequences and the PRE. We note that the affinity of SF2/ASF for any of the $d s x$ RE constructs is somewhat less than that of any of the other proteins tested $(20-60 \%$ bound with $1.5 \mu \mathrm{M}$ SC35 versus $5-20 \%$ bound with $1.5 \mu \mathrm{M}$ SF2). These small differences in affinity may explain why SC35 and SRp55, but not SF2/ASF, are able to form a committed regulatory complex with Tra and Tra2 in vitro (Tian and Maniatis 1993).

Both the dsx repeats and the PRE are required for cooperative binding of Tra, Tra2, and SR proteins to the dsxRE

We also determined the binding specificities of Tra, Tra2, and the SR proteins to dsXRE constructs containing (R2-5PRE) or lacking (R2-5) the PRE (Fig. 5A). Binding specificity was measured by performance of binding reactions in the presence of increasing amounts of either specific or nonspecific unlabeled RNA competitor. The binding specificity is calculated as the ratio of the amount of nonspecific versus specific competitor required for a 50\% decrease in binding (see Fig. 5B for representative graphs). Consistent with previous studies, Tra2 bound to the R2-5PRE RNA with significant specificity (Hedley and Maniatis 1991; Tian and Maniatis 1992). In contrast, the SR proteins bound with little, although measurable, specificity. Tra also bound to R25PRE with some specificity, although less than that of Tra2. Previous results had indicated that Tra did not bind specifically to the $d s x R E$ (Tian and Maniatis 1992). This apparent contradiction is explained by the fact that the nitrocellulose binding assay is more quantitative than the cross-linking assays used previously. However, it is important to note that the relative specificities of single and multiple proteins determined by filter binding are all qualitatively consistent with cross-linking studies (data not shown).

Interestingly, the specificity of binding of the combination of Tra and an SR protein is 10-15 times greater than that observed with either protein alone. Moreover, the addition of Tra2 to Tra plus either SC35 or SRp55 further increases the specificity of the Tra + SR complex by two- to threefold. This observation is consistent with previous results showing that levels of SR proteins bound to the $d s \times R E$ increase upon addition of Tra and Tra2 (Tian and Maniatis 1993, 1994) and strongly sug- gests that these proteins bind cooperatively to the dsxRE.

A comparison of the binding specificities with the R2-5 RNA and the R2-5PRE reveals interesting differences. Consistent with the observed difference in binding affinities, the specificity of Tra2 decreased fivefold upon removal of the PRE. However, the specificity of Tra, SC35 and SRp55 remains essentially unchanged. The specificity of SF2/ASF for R2-5 could not be measured because the binding affinity of this protein is so low. Furthermore, although the combination of Tra + SC35 or SRp55 showed a marked increase in specificity for R2-5 over the specificity of either protein alone, this specificity was not further increased upon addition of Tra2. Thus the specificity of Tra2 alone, as well as its ability to bind along with Tra $+S R$, is significantly decreased in the absence of the PRE (see Fig. $5 \mathrm{~B}, \mathrm{Cl}$.

We note that SF2/ASF behaves differently from SC35 and SRp55 in these binding specificity studies. SF2/ASF appears to interact with Tra on the R2-5PRE RNA, but Tra2 does not further stimulate the binding. This observation is also consistent with the fact that SF2/ASF does not form a committed regulatory complex with Tra and Tra2 (Tian and Maniatis 1993). The binding of Tra + SF2/ ASF to the R2-5 RNA is the same as the binding of Tra alone. The addition of Tra2 does have a slight effect; however, the specificity is still less than any other proteins in combination. Thus we believe the effect of Tra2 addition to Tra + SF2 is unlikely to be a result of significant interactions among the three proteins.

Finally, we determined whether Tra, Tra2 and the SR proteins could bind directly to the PRE in isolation. Binding specificity measurements were made as described above, with the PRE as probe and a dimer of the repeat sequence as nonspecific competitor. This nonspecific competitor was used because it is of similar length to the isolated PRE, it contains a low purine content, and none of the proteins tested had any measurable specificity for even a repeat sequence tetramer (data not shown).

As shown in Table 1, Tra2 binds to the isolated PRE with 15-fold specificity. This is less than the specificity of Tra2 for R2-5PRE but at least threefold more than the

Table 1. Binding specificities of Tra, Tra2, and $S R$ proteins for the PRE

\begin{tabular}{lc}
\hline \multicolumn{2}{c}{ Specificity for isolated PRE } \\
\hline protein & specificity \\
\hline Tra2 & 15 \\
Tra & 3 \\
SC35 & 2 \\
SF2/ASF & 5
\end{tabular}

Average binding specificities are based on at least three independent experiments. As a result of inherent error, standard deviations of all reported averages are between $10 \%$ and $30 \%$; therefore, all differences of at least twofold are considered significant. 
specificity of Tra2 for R2-5. In contrast, the specificity of Tra for the isolated PRE is fourfold less than its specificity for either R2-5PRE or R2-5. Furthermore, SC35 binds to the isolated PRE with little or no specificity, whereas SF2 binds to the PRE alone with as much specificity as to R2-5PRE. These data are consistent with the idea that SF2 and Tra2 bind primarily to the PRE, whereas the PRE is not involved in the binding of Tra and SC35 to the $d s x R E$. The difference in the specificity of Tra2 for the R2-5PRE construct versus the isolated PRE may be due to a lack of defined secondary structure of the isolated PRE. Alternatively, the difference could reflect a minor component of Tra2 binding that is dependent on the repeat sequences.

\section{Discussion}

Previous studies demonstrated that Tra- and Tra2-dependent female-specific splicing of $d s x$ pre-mRNA requires the repeat sequences located in the $d s x R E$ (Hedley and Maniatis 1991; Hoshijima et al. 1991; Ryner and Baker 1991; Tian and Maniatis 1992). We show here that an additional enhancer motif located within the $d s x \mathrm{RE}$, the PRE, is also essential for normal levels of Tra/Tra2-dependent splicing enhancer activity. Moreover, we show that the PRE is required for normal levels of Tra2 binding to the $d s x \mathrm{RE}$.

\section{The $\mathrm{ds} \times R E$ contains two distinct enhancer elements}

The PRE is essentially identical to previously defined purine-rich exonic enhancers, whereas the $d s x$ repeat sequences are distinct. Interestingly, both of these sequences are independently able to constitutively activate splicing when placed in close proximity $(100$ nucleotides/ to the weak female-specific $3^{\prime}$ splice site. A third type of sequence capable of activating the $d s x$ femalespecific $3^{\prime}$ splice site was identified in an attempt to inactivate a purine-rich enhancer by extensive base substitutions (Staknis and Reed 1994). This pyrimidine-rich sequence is active, and UV cross-linking studies revealed a switch in SR protein binding to the wild-type and mutant sequences. SRp40 was shown to bind to the purinerich sequence, whereas the pyrimidine-rich sequence was recognized primarily by SRp30 (Staknis and Reed 1994).

On the basis of the observation that SR proteins bind specifically to the $d s x R E$ in the absence of Tra and Tra2, it was proposed that the constitutive activity of this enhancer is mediated by SR proteins (Tian and Maniatis 1994). Here we show that deleting the PRE does not decrease the affinity of SC35 for the $d s x \mathrm{RE}$ nor does it affect its constitutive activity of the enhancer. Thus the repeat sequences alone may be sufficient for recognition by $\mathrm{SC} 35$, and the constitutive activity of the R2-5 and $\mathrm{R} 2 \times 4$ elements may therefore require SC35. In contrast, the binding of SF2/ASF to the dsxRE was dramatically decreased by deletion of the PRE. Thus SF2/ASF may be required for the constitutive splicing enhancer activity of the PRE. This possibility is consistent with the fact that SF2/ASF has been shown to bind to other purinerich splicing enhancers (Sun et al. 1993b), and that SF2/ ASF binds to the isolated PRE with fivefold specificity.

\section{Tra/Tra2-regulated activation of splicing requires both} enhancer elements

When the $d s x \mathrm{RE}$ is distant (300 nucleotides) from the female-specific $3^{\prime}$ splice site, it is no longer able to constitutively activate splicing, but instead requires the activity of Tra and Tra2 (Tian and Maniatis 1994). Although either the repeat sequences or the PRE can constitutively activate splicing, neither alone is sufficient to mediate the normal Tra/Tra2-dependent activation of splicing. Low levels of splicing activity can be detected when the PRE is removed from the dsxRE, but at least five times as much Tra and Tra2 is required to observe splicing activity. When fewer repeat sequences are present, the requirement for the PRE is even greater.

Contrary to the in vitro results reported here, a dimer of the consensus repeat sequence located 300 nucleotides from the female-specific $3^{\prime}$ splice site was shown to be sufficient for Tra/Tra2-dependent female-specific splicing of $d s x$ in vivo (Inoue et al. 1992). This difference could be due to the fact that the latter experiments were carried out in cultured Drosophila cells where the efficiency of splicing would be expected to be greater than that observed in the in vitro system used in our studies. Alternatively, additional factors that compensate for the loss of the PRE may be present in the cultured Drosophila cells.

The PRE is required for Tra/Tra2-dependent splicing and binding of Tra2

Tra and Tra2 play a critical role in the assembly of a multiprotein regulatory complex on the dsxRE. This complex includes SR proteins and possibly other splicing factors (Tian and Maniatis 1993, 1994). In this paper, we provide direct evidence that the assembly of this complex involves cooperative interactions between Tra and SR proteins and between a complex of these two proteins and Tra2. We also demonstrate that deletion of the PRE decreases the specificity of Tra2 binding to the $d s \times \mathrm{RE}$ and eliminates the cooperative binding between Tra2 and the Tra/SR protein complex. Moreover, we show that Tra2 binds specifically to the isolated PRE. These observations suggest that the PRE functions as a high affinity Tra2-binding site that is required for cooperative interactions with Tra and SR proteins, and that this cooperativity is required for the assembly of the multicomponent splicing enhancer complex. This possibility is consistent with the observations that both Tra and Tra2 can interact with each other and with SR proteins, and that SR proteins interact with each other $(\mathrm{Wu}$ and $\mathrm{Ma}$ niatis 1993; Amrein et al. 1994). The cooperative binding of proteins containing SR domains may also have more general implications regarding mechanisms of splice site selection. Although individual SR proteins bind to RNA with low specificity /Caceres and Krainer 1993; Zuo and 
Manley 1993), cooperative interactions between different SR proteins could lead to the formation of highly specific RNA-protein complexes on pre-mRNA. Thus, the diversity of RNA sequences recognized by heteromultimers of SR proteins could be enormous.

Two previous reports have shown that Tra2 binds to isolated repeat sequences (Hedley and Maniatis 1991; Inoue et al. 1992). In one study Tra2 was shown to bind to a dimer of the repeat consensus sequence, but not to a repeat sequence containing point mutations that inactivate the element in vivo. However, the affinity of Tra2 for the synthetic repeats was not compared with that for the intact $d s x$ RE (Inoue et al. 1992). In the other study Tra2 was shown to bind weakly to a multimerized repeat sequence, but binding to such a construct was at least 10 -fold less than the binding of Tra2 to a construct containing repeats 2-6 (inclusive of the PRE) (Hedley and Maniatis 1991). Furthermore, we have found that neither Tra nor Tra2 bind specifically to the $\mathrm{R} 2 \times 4$ repeat in filter-binding experiments (data not shown).

On the basis of the results presented here we propose that the complexity of the $d s x$ RE may be a general characteristic of regulated splicing enhancers. In the case of constitutive enhancers, which act in close proximity to the $3^{\prime}$ splice site, a single type of element may be sufficient for their activity. By contrast, the ability of the regulated enhancer to function at a distance in response to specific regulatory proteins may require a more complex organization. Like regulated transcriptional enhancers (Tijan and Maniatis 1994), these splicing enhancers may require synergistic interactions between multiple protein components which combine to achieve a high level of specificity and stability of the regulatory complex.

\section{Materials and methods}

\section{RNA}

The splicing substrates in which the $d s x \mathrm{RE}$ is located $100 \mathrm{nu}$ cleotides downstream from the female-specific $3^{\prime}$ splice site were derived from the D16 plasmid (Tian and Maniatis 1994), which contains 84 nucleotides of $d s x$ exon 3 , the complete 114 nucleotide intron between exons 3 and 4 , and the first $70 \mathrm{nu}$ cleotides of the fourth exon followed by polylinker sequence. D16 linearized with $B a m H I$ was used to synthesize the $d s x \Delta \mathrm{E}$ transcript. Construct R2-5PRE was generated by subcloning an MluI-FspI fragment of the $d s x \mathrm{RE}$ into the polylinker of D16 and linearizing with $D r a l$ for in vitro transcription. R2-5 contains a $d s x R E$ fragment beginning at MluI and ending at a PCR-generated $B a m H I$ site between the fifth repeat and the PRE. R $2 \times 4$ contains four copies of the repeat containing sequence GGATCCGTCTTCAATCAACATACGCGAGATCT multimerized into the BamHI to BglII site of D16. The $d s x$ PRE construct was also generated by insertion of the PRE-containing oligo GGATCCAAAGGACAAAGGACAAAATGTAGATCT into the BamHI/BglII sites of D16.

The splicing substrates in which the $d s x$ RE sequences are placed 300 nucleotides away from the 3 ' splice site were generated from plasmid D1 (Tian and Maniatis 1992), which contains the entire wild-type $d s x$ fourth exon. When linearized with DraI, this construct is the template for the R1-5PRE transcript.
To facilitate subcloning, the polymerase chain reaction (PCR) was used to introduce an EcoRI site just upstream of the first repeat sequence in the D1 construct. R1-5 was derived by substitution of an EcoRI-BamHI fragment of the $d s x$ RE (by use of the previously described PCR-generated sites) for the fourth exon fragment downstream of the EcoRI site in D1. Inserts R25PRE, R2-5, R2x4, and $d s \times$ PRE were also subcloned into this EcoRI site in Dl. The construct R1-5mPRE was derived from R1-5PRE by PCR mutagenesis to change the sequence of the PRE from AAAGGACAAA $\underline{A G G A C A A A A}$ to AATCGACEATCGACCAAA.

The probes R2-5PRE and R2-5 used for binding studies correspond to inserts R2-5PRE and R2-5 subcloned directly into SP73 for in vitro transcription. Binding probe R2-6 was generated by linearization of the MluI-FspI R2-5PRE construct downstream of the FspI site. The nonspecific competitor was transcribed from construct D6 (Tian and Maniatis 1994) linearized with ApaLI. The isolated PRE probe for binding studies was generated by removal of the $d s x$ exon and intron sequences from the $d s \times$ PRE construct. The nonspecific competitor, $\mathrm{R} 2 \times 2$, was transcribed from an SP72 construct containing a dimer of the repeat sequence oligo described above. None of the probes or competitors used for binding studies contained any splicing sites.

Splicing substrates and binding probes were uniformly labeled with $\left|{ }^{32} \mathrm{P}\right| \mathrm{UTP}$, with a $1: 18$ molar ratio of $\left[{ }^{32} \mathrm{P} \mid \mathrm{UTP}\right.$ to cold UTP. Competitors for binding studies were transcribed without the addition of any labeled nucleotides.

In vitro splicing reactions

In vitro splicing reactions werc carried out as described in Tian and Maniatis (1992).

\section{Recombinant proteins}

Recombinant baculoviruses expressing Tra and Tra2, and purification of Tra and Tra2 were as described in Tian and Maniatis (1992). Recombinant viruses expressing SC35, SF2/ASF, and SRp55, and purification of these proteins were as described in Tian and Maniatis (1993).

Nitrocellulose filter binding assays

Binding reactions to be analyzed by filtration were done in 145 $\mathrm{mm} \mathrm{KCl}, 3.2 \mathrm{~mm} \mathrm{MgCl}, 1 \mathrm{~mm}$ ATP with $50 \mu \mathrm{g} / \mathrm{ml} \mathrm{BSA}$ and 0.5 $\mathrm{mg} / \mathrm{ml}$ yeast tRNA in a total volume of $10 \mu \mathrm{l}$. Probe concentration was $\sim 1 \mathrm{nM}$ in all reactions. For binding specificity experiments the amount of protein used was in the linear range of the binding curve whenever possible. Binding reactions were incubated at $30^{\circ} \mathrm{C}$ for $20 \mathrm{~min}$, and then returned to ice. Reactions were diluted before loading with $90 \mu \mathrm{l}$ of buffer containing $145 \mathrm{mM} \mathrm{KCl}$ and $3.2 \mathrm{mM} \mathrm{MgCl}_{2}$ at $4^{\circ} \mathrm{C}$, and then filtered immediately through a $0.45 \mu \mathrm{M}$ pore nitrocellulose filter sandwiched in a filtration minifold (Schleicher \& Schuell). Dilution was done to ensure that reactions were loaded evenly on filters. Filters were washed once with $200 \mu$ l of dilution buffer, then the vacuum was broken and the filters allowed to air dry briefly. Retention of labeled RNA on filters was analyzed by liquid scintillation. Percentage of RNA bound to filters relative to input RNA was corrected for retention of probe in the absence of protein. For all of the reported experiments this background retention was $<3 \%$ of input RNA.

\section{Acknowledgments}

We thank M. Tian for providing nuclear extracts and several of the $d s x$ constructs. We also thank members of the Maniatis lab 
for critical comments on the manuscript. This work was funded by a grant from the National Institutes of Health to T.M.

The publication costs of this article were defrayed in part by payment of page charges. This article must therefore be hereby marked "advertisement" in accordance with 18 USC section 1734 solely to indicate this fact.

\section{References}

Amrein, H., M. Gorman, and R. Nöthiger. 1988. The sex-determining gene tra-2 of Drosophila encodes a putative RNA binding protein. Cell 55: 1025-1035.

Amrein, H., M.L. Hedley, and T. Maniatis. 1994. The role of specific protein-RNA and protein-protein interactions in positive and negative control of pre-mRNA splicing by Transformer 2. Cell 76: 735-746.

Baker, B.S. 1989. Sex in flies: The splice of life. Nature 340: 521-524.

Boggs, R.T., P. Gregor, S. Idriss, J.M. Belote, and M. McKeown. 1987. Regulation of sexual differentiation in D. melanogaster via alternative splicing of RNA from the transformer gene. Cell 50: 739-747.

Burtis, K.C. and B.S. Baker. 1989. Drosophila doublesex gene controls somatic sexual differentiation by producing alternative spliced mRNAs encoding related sex-specific polypeptides. Cell 56: 997-1010.

Caceres, J.F. and A.R. Krainer. 1993. Functional analysis of premRNA splicing factor SF2/ASF structural domains. EMBO /. 12: 4715-4726.

Dirksen, W.P., R.K. Hampson, Q. Sun, and F.M. Rottman. 1994. A purine-rich exon sequence enhances alternative splicing of bovine growth hormone pre-mRNA. I. Biol. Chem. 269: 6431-6436.

Fu, X.-D. and T. Maniatis. 1992. Isolation of a complementary DNA that encodes the mammalian splicing factor SC35. Science 256: $535-538$.

Hedley, M.L. and T. Maniatis. 1991. Sex-specific splicing and polyadenylation of $d s x$ pre-mRNA requires a sequence that binds specifically to tra-2 protein in vitro. Cell 65: 579-586.

Horowitz, D.S. and A.R. Krainer. 1994. Mechanisms for selecting $5^{\prime}$ splice sites in mammalian pre-mRNA splicing. Trends Genet. 10: 100-106.

Hoshijima, K., K. Inoue, I. Higuchi, H. Sakamoto, and Y. Shimura. 1991. Control of doublesex alternative splicing by transformer and transformer-2 in Drosophila. Science 252: 833-836.

Inoue, K., K. Hoshijima, I. Higuchi, H. Sakamoto, and Y. Shimura. 1992. Binding of the Drosophila Transformer and Transformer-2 proteins to the regulatory elements of doublesex primary transcripts for sex-specific RNA processing. Proc. Natl. Acad. Sci. 89: 8092-8096.

Krainer, A.R., A. Mayeda, K. Kozak, and G. Binns. 1991. Functional expression of clones of human splicing factor SF2: Homology to RNA binding proteins, U1 70K, and Drosophila splicing regulators. Cell 66: 383-394.

Lamm, G.M. and A. Lamond. 1993. Non-snRNP protein splicing factors. Biochim. Biophys. Acta 1173: 247-265.

Lavigueur, H., L. Branche, A.R. Kornblihtt, and B. Chabot. 1993. A splicing enhancer in the human fibronectin alternate ED1 exon interacts with SR proteins and stimulates U2 snRNP binding. Genes \& Dev. 7: 2405-2417.

Maniatis, T. 1991. Mechanisms of alternative pre-mRNA splicing. Science 251: 33-34.

McKeown, M., J.M. Belote, and R.T. Boggs. 1988. Ectopic expression of the female transformer gene product leads to female differentiation of chromosomally male Drosophila. Cell 53: 887-895.

Nagoshi, R.N. and B.S. Baker. 1990. Regulation of sex-specific RNA splicing at the Drosophila doublesex gene: cis-acting mutations in exon sequences alter sex-specific RNA splicing patterns. Genes \& Dev. 4: 89-97.

Nagoshi, R.N., M. McKeown, K.C. Burtis, I.M. Belote, and B.S. Baker. 1988. The control of alternative splicing at genes regulating sexual differentiation in D. melanogaster. Cell 53: 229-236.

Rio, D. 1992. RNA binding proteins, splice site selection and alternative pre-mRNA splicing. Gene Exp. 2: 1-5.

Ryner, L.C. and B.S. Baker. 1991. Regulation of doublesex premRNA processing occurs by 3 '-splice site activation. Genes \& Dev. 5: 2071-2085.

Staknis, D. and R. Reed. 1994. SR proteins promote the first specific recognition of pre-mRNA and are present together with the Ul small nuclear ribonucleoprotein particle in a general splicing enhancer complex. Mol. Cell. Biol. 14: 7670-7682.

Steinmann-Zwicky, M., H. Amrein, and R. Nöthiger. 1990. Genetic control of sex determination in Drosophila. $A d v$. Genet. 27: 189-237.

Sun, Q., R.K. Hampson, and F.M. Rottman. 1993a. In vitro analysis of bovine growth hormone pre-mRNA alternative splicing. J. Biol. Chem. 268: 15659-15666.

Sun, Q., A. Mayeda, R.K. Hampson, A.R. Krainer, and F.M. Rottman. 1993b. General splicing factor SF2/ASF promotes alternative splicing by binding to an exonic splicing enhancer. Genes \& Dev. 7: 2598-2608.

Tanaka, K., A. Watakabe, and Y. Shimura. 1994. Polypurine sequences within a downstream exon function as a splicing enhancer. Mol. Cell. Biol. 14: 1347-1354.

Tian, M. and T. Maniatis. 1992. Positive control of pre-mRNA splicing in vitro. Science 256: 237-240.

__ 1993. A splicing enhancer complex controls alternative splicing of doublesex pre-mRNA. Cell 74: 105-114.

-1994. A splicing enhancer exhibits both constitutive and regulated activities. Genes \& Dev. 8: 1703-1712.

Tjian, R. and T. Maniatis. 1994. Transcriptional activation: A complex puzzle with few easy pieces. Cell 77: 5-8.

Watakabe, A., K. Tanaka, and Y. Shimura. 1993. The role of exon sequences in splice site selection. Genes \& Dev. 7: 407-418.

Wu, J.Y. and T. Maniatis. 1993. Specific interactions between proteins implicated in splice site selection and regulated alternative splicing. Cell 75: 1061-1070.

$\mathrm{Xu}, \mathrm{R} ., \mathrm{J}$. Teng, and T.A. Cooper. 1993. The cardiac troponin T alternative exon contains a novel purine-rich positive splicing element. Mol. Cell. Biol. 13: 3660-3674.

Zahler, A.M., S.W. Lane, J.A. Stolk, and M.B. Roth. 1992. SR proteins: A conserved family of pre-mRNA splicing factors. Genes \& Dev. 6: 837-847.

Zuo, P. and J.L. Manley. 1993. Functional domains of the human splicing factor ASF/SF2. EMBO I. 12: 4727-4737. 


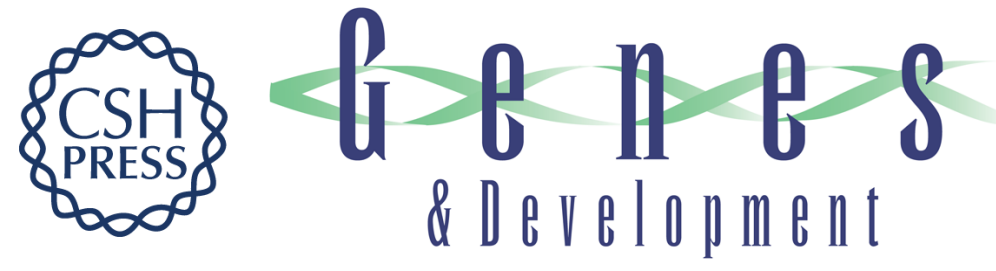

\section{Synergistic interactions between two distinct elements of a regulated splicing enhancer.}

$\mathrm{K}$ W Lynch and T Maniatis

Genes Dev. 1995, 9:

Access the most recent version at doi:10.1101/gad.9.3.284

References This article cites 35 articles, 17 of which can be accessed free at:

http://genesdev.cshlp.org/content/9/3/284.full.html\#ref-list-1

License

Email Alerting

Service

Receive free email alerts when new articles cite this article - sign up in the box at the top right corner of the article or click here.

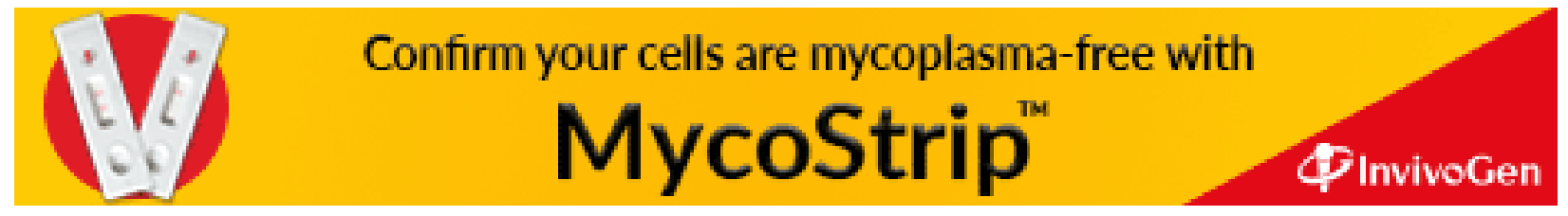

